\title{
Aplicação da laserterapia de baixa intensidade na odontologia: revisão integrativa
}

\author{
Application of low intensity laser therapy in dentistry: integrative review
}

Aplicación de la terapia láser de baja intensidad en odontología: revisón integrativa

José Milton de Aquino e Silva Neto ${ }^{1 *}$, Júllian Karen Bezerra dos Santos ${ }^{1}$, Natália Mirelle Araújo Gomes $^{1}$, Clayton Clenisson de Carvalho Silva ${ }^{1}$, João Vitor Brasil Alves Rodrigues Almeida Barros ${ }^{1}$, Michelle Leão Bittencourt Brandão Medeiros ${ }^{1}$.

\section{RESUMO}

Objetivo: Este trabalho tem como objetivo realizar uma revisão integrativa para analisar as indicações do tratamento do laser de baixa intensidade Low Level Laser Therapy (LLLT) no uso odontológico. Métodos: Como estratégia de busca foi estabelecido pesquisas nas bases de dados PubMed/MedLine, LiLacs e SciELo, no período de 2014-2019, combinando os descritores "laserterapia", "laserterapia na odontologia" e "low level laser therapy e dentistry" aos descritores booleanos nas respectivas fontes de pesquisa. Resultados: $O$ laser de baixa intensidade vem sendo utilizado para fins terapêuticos na odontologia, seus efeitos possibilitam seguimentos benéficos nas perspectivas de observação clínica e funcional, minimizando o quadro doloroso através do seu efeito analgésico, antiinflamatório e bioestimulante, otimizando dessa forma o processo de cicatrização. Considerações Finais: A aplicação do laser de baixa intensidade é um procedimento feito com muita frequência por profissionais da área odontológica, onde possui grande eficácia nos procedimentos coadjuvantes aos convencionais, sendo o tratamento bastante confiável, onde não possui efeitos colaterais decorrentes, porém os profissionais devem estar capacitados para a realização de um procedimento satisfatório.

Palavras-chave: Terapia a laser, Terapia com luz de baixa intensidade, Radiação, Radiação eletromagnética.

\section{ABSTRACT}

Objective: This paper aims to conduct na integrative review to analyse the indications of low-level laser therapy (LLLT) in dental use. Methods: As a search strategy, searches in the PubMed/MedLine, LiLacs and SciELo databases were established in the 2014-2019 period, combining the descriptors "laser therapy", "laser therapy in dentistry" and "low level laser therapy and dentistry" with Boolean descriptors. In their search sources. Results: The low intensity laser has been used for therapeutic purposes in dentistry, its effects enable beneficial follow-up in the perspectives of clinical and functional observation, minimizing the painful picture through its analgesic, anti-inflammatory and biostimulant effect, thus optimizing the healing process. Final Considerations: The application of low intensity laser is a procedure very often done by dental professionals, where it is very effective in supporting the conventional procedures, and the treatment is very reliable, where there are no side effects, but professionals should be able to performing a satisfactory procedure.

Key Words: Laser therapy, Low-level light therapy, Radiation, Electromagnetic radiation.

${ }^{1}$ Centro Universitário CESMAC (CESMAC), Maceió-Alagoas.

*E-mail: Milton_neto_166@hotmail.com

SUBMETIDO EM: 10/2019

ACEITO EM: 11/2019

PUBLICADO EM: 1/2020 


\section{RESUMEN}

Objetivo: Este artículo tiene como objetivo realizar una revisión integradora para analizar las indicaciones de la terapia con láser de bajo nivel (LLLT) en el uso dental. Métodos: Como estrategia de búsqueda, se realizaron búsquedas en las bases de datos PubMed/MedLine, LiLacs y SciELo, de 2014-2019, combinando los descriptores "terapia láser", "Terapia láser em odontología" y "terapia láser y odontología de bajo nivel" com los descriptores booleanos. Em sus fuentes de búsqueda. Resultados: El láser de baja intensidad se ha utilizado con fines terapéuticos en odontología, sus efectos permiten un seguimiento beneficioso en las perspectivas de observación clínica y funcional, minimizando la imagen dolorosa a través de su efecto analgésico, antiinflamatorio y bioestimulante, optimizando así el proceso de curación. Consideraciones finales: La aplicación de láser de baja intensidad es un procedimiento muy a menudo realizado por profesionales dentales, donde es muy efectivo para apovar los procedimientos convencionales, y el tratamiento es muy confiable, donde no hay efectos secundarios, pero los profesionales deberían poder realizar um procedimiento satisfactorio.

Palabras Clave: Terapia por láser, Terapia por luz de baja intensidad, Radiación, Radiación electromagnética.

\section{INTRODUÇÃO}

Nos últimos anos o desenvolvimento tecnológico, especialmente os estabelecidos por meio de procedimentos físicos, tem colaborado com a melhoria da tecnologia principalmente os que são advindos das áreas de diagnósticos e terapias. Um dos avanços encontrados nessa área é constituído pela laserterapia, sendo constituída por um laser que é acrônimo da língua inglesa (Amplificação de Luz por Emissão Estimulada de Radiação) que é uma luz bastante desenvolvida, onde possui várias aplicabilidades em todas as áreas de estudos, sendo inclusa no campo da saúde como uma ferramenta bastante significativa principalmente em procedimentos, como é o caso das especializações odontologias (SRIVASTAVA VK e MAHAJAN S, 2014).

A luz encontrada no laser expressa peculiaridades bastante significativas e particulares que as diferenciam das luzes convencionais incandescentes, o que estabelece viabilidades nas atuações dos Cirurgiões Dentistas (CD). A luz encontrada no laser apresenta radiações encontradas nos espectros de luzes que diferenciam das ultravioletas aos infravermelhos, sendo percorrida pelo espectro visível. Os processos de radiações não apresentam aspectos invasivos sendo aceita entre os tecidos (POL R, et al., 2016).

Existem dois tipos de laser utilizados na área da saúde, os que apresentam grande intensidade de luz irradiada sendo esses geralmente mais utilizados em procedimentos cirúrgicos conservadores, onde possui como objetivo a diminuição da dor no pós-cirúrgico, e o laser de pequena intensidade (LLLT), onde visa o estabelecimento terapêutico, proporcionando analgesia, cicatrização, estimulo de biomodulação dos tecidos e efeitos anti-inflamatório, além disso, possui características benéficas em terapias fotodinâmicas no momento que é relacionada aos agentes responsáveis pela fotossensíbilidade acarretando o melhor tratamento de infecção (ANG KHAW CM, et al., 2018).

Os lasers de alta intensidade são bastante utilizados nos Estados Unidos, porém no Brasil ele possui pouca aplicação pelos profissionais, isso por causa do elevado custo quando comparado com o LLLT (CIEPLIK F, et al., 2018). Os lasers que possuem baixa intensidade são mais acessíveis devido seu custo e benefício, onde por sua vez são cruciais para os profissionais da odontologia visto que apresentam inúmeras formas de tratamentos nas respectivas especialidades da área, sendo uma forma segura, porém não farmacológica, que melhoram os tratamentos metabólicos mediante o processo de incorporação e fatores energéticos realizados pelos cromóforos, dos quais ocasionam modificações no aspecto funcional das mitocôndrias, e de modo consequente, mudança respiratória da célula, com maior produção de adenosina trifosfato (ATP), estabelecendo a ativação de espécies reagentes de oxigênio intracelular, resultando na multiplicação de fibroblastos, cadeias de colágenos e melhora nas respostas de eventuais inflamações resultando na melhor reparação do tecido (SANT'ANNA E F, et al., 2017). 
Constantemente, devido a desinformação das interações correlacionadas entre o laser e os tecidos, e consequentemente os efeitos terapêuticos, as radiações de ondas eletromagnéticas não ionizantes aplicadas nas mais diversas formas clinicas e até mesmo a utilização do próprio aparelho, vários Cirurgiões Dentistas não utilizam, deixando assim de melhorar os resultados finais dos procedimentos realizados nos pacientes (SANT'ANNA EF, et al., 2017).

Com isso, esse estudo possuiu como objetivo analisar as possíveis indicações da laserterapia de baixa intensidade visto que essa ferramenta está cada dia mais presente no cotidiano do Cirurgião Dentista nas mais diversas áreas da atuação com aspectos modernos, buscando sempre o melhoramento na implantação de casa especialização.

\section{METODOLOGIA}

Esta pesquisa constituiu-se através de uma revisão integrativa realizada entre os períodos de março de 2019 a outubro de 2019, onde foram analisados artigos publicados em bases de dados eletrônicos Literatura Latino Americana e do Caribe em Ciências da Saúde (LILACS), Literatura Internacional em Ciência da Saúde (MEDLINE) e Scientific Electronic Library Online (SCIELO) e livros que estavam disponíveis nos acervos bibliográficos presentes na biblioteca central do Centro Universitário Centro de Ensino Superior de Maceió (CESMAC).

Para tanto o estudo foi estabelecido através dos seguintes critérios: os desenhos dos estudos deveriam ser ensaios clínicos controlados randomizados em humanos, no idioma inglês, escolhidos os trabalhos publicados nos últimos 6 anos (2014 a 2019) que se referiram a aplicação de laserterapia no consultório Odontológico, tendo como critérios de exclusão os períodos de publicações, relatos de casos, séries de casos, estudos em animais, ensaios não controlados, artigos de revisão, monografias e os artigos que não possuíam relevância com a temática, sendo selecionados os textos científicos que apresentavam na íntegra o real objetivo do trabalho, observando a importância da diferenciação dos respectivos lasers utilizados para cada área odontológica visando o melhor procedimento para a obtenção de resultados satisfatórios e benéficos para o paciente. Os descritores utilizados foram: Terapia a laser, Terapia com luz de baixa intensidade, Radiação, Radiação eletromagnética.

Os artigos foram estabelecidos por 6 pesquisadores: primeiramente foram feitas as avaliações da temática e posteriormente, a leitura crítica do resumo para a exploração dos discernimentos de exclusão. Logo em seguida foram estabelecidos a leitura de todos eles, dos quais se extraíram os subsídios de interesse, como também foram averiguadas suas listas de referências, visando localizar artigos complementares para o enriquecimento científico da revisão. Com os artigos escolhidos, foram tabuladas as centrais variáveis de instância, como a dimensão da amostra, o objetivo, a metodologia, os parâmetros analisados e os fundamentais resultados.

\section{RESULTADOS E DISCUSSÃO}

A procura por condutas que minimizem determinadas dores de uma forma geral não é um afligimento decorrente da sociedade moderna, desde o início das civilizações existiam buscas incessantes de meios que diminuíssem as dores, a luz solar quando entrava em contato com algumas ervas que eram colocadas na pele possuíam a otimização dos efeitos terapêuticos. Da mesma maneira que os desenvolvimentos dolorosos ocasionam vários processos que incomodam, sendo eles psicológicos como também podendo ser patológicos, assim sendo, a incessante demanda por recursos terapêuticos eficientes que visam minimizar ou até mesmo acabar com processos dolorosos melhorando o bem estar pessoal são analisados e aprimorados nos dias atuais, sendo utilizado o laser como mediador de tal estudo (SHIMTOME LK, et al., 2007).

Os povos asiáticos desde 1400 a.C. recorriam a óleos fotossensibilizantes extraídos de plantas que eram ativados através da absorção da luz do sol buscando possuir um tratamento para o vertiligo. Apesar de não se saber ao certo quando surgiu a criação do laser, é entendido que o processo de desempenho ocorrido por 
esses instrumentos que possuem emissão de luz afamados por lasers são de suma relevância nos dias de hoje. Os primeiros estudos na área odontológica que utilizaram o laser do tipo rubi foram feitos "in vitro" esse estudo possibilitou a obtenção de maior concentração de temperatura no local onde foi exposto a luz, tendo como resultado a queima dos tecidos (SHIMTOME LK, et al., 2007).

Os estudos feitos com o objetivo da utilização do laser na odontologia deu-se através de pesquisas relacionadas as ondas de luzes que eles propagavam, sendo observado os que possuíam feixes de luzes com baixa intensidade possibilitavam resultados mais satisfatórios, pois foi constatado que suas luzes monocromáticas e bastante focada são capazes de penetrar em camadas mais profundas da pele ocasionando a estimulação dos fotorreceptores presentes, acarretando por sua vez melhores atividades funcionais e metabólicas do sistema celular possibilitando resultados satisfatórios como: efeitos analgésicos, antiinflamatórios e de bioestimulações (TORKZABAN P, et al., 2018).

O LLLT apresenta como sendo umas das principais características no uso odontológico a prevenção e tratamento das afecções orofaciais, como: processos relacionados a mucosites, aftas, sensibilidades nos procedimentos restauradores, na disfunção temporo mandibulares, nevralgias, xerostomias, pericoronarites, alveolites, osteoradionecroses, e pós processos cirúrgicos através dos seus efeitos analgésicos, antiflamatórios e biomodulação tecidual (FARIAS RD, et al., 2016).

O processo de aplicação do LLLT decorre significantemente na diminuição ou até mesmo na eliminação da dor, esses resultados bastante representativos fez com quer nos últimos anos os pacientes que sofrem com alguma dor crônica ou aguda busquem os Cirurgiões Dentistas, um dos grandes motivos que levam eles aos consultórios são as sensibilidades dos elementos dentários, problema esse que afeta grande parte da sociedade, ocasionando grande desconforto nos tecidos duros e moles que compõem o dentes, do qual as camadas que revestem os túbulos da dentina ficam expostos por causa do desgaste do esmalte dental. Com a utilização do laser ocorre o processo excitatório das terminações nervosas ocasionando a diminuição da dor, proporcionando um efeito terapêutico bioestimulante, biorregulador, antinflamatório e por fim cicatrizante (NABI S, et al., 2018).

Por tratar-se de uma técnica minimamente agressiva, acessível, simples e não traumática, onde a literatura não demostra relatos de toxicidade, a utilização do laser é alegórica nos respectivos procedimentos que visem os procedimentos terapêuticos de tecidos sem possuir risco de prejudicar as células saudáveis. Ao se proceder as determinadas patologias, conseguirá utilizar o Laser vermelho, infravermelho ou ambos em conjunto, dependendo das particularidades de cada caso. A laserterapia LLLT vem sendo usada há mais de 5 décadas porém, não se possui uma real conformidade a respeito de um protocolo uniformizado para a aplicação clínica pelos Cirurgiões Dentistas (CD), é necessário um curso preparatório para esses profissionais buscando deixá-los qualificados, sendo levado em consideração as alterações dos parâmetros que podem ser aplicados em comprimento de onda para cada especificidade, a energia que será utilizada para determinado procedimento, fluência da utilização, potência do laser a ser utilizado, tempo de tratamento e eventuais repetição (TORKZABAN P, et al., 2018).

De acordo com as análises e resultados da pesquisa do presente trabalho, foram selecionados 10 artigos mais recentes e mais utilizados na área odontológica onde foram discutidos na luz do entendimento da importância da aplicação da leserterapia nas mais diversas áreas de especializações odontológicas enfatizando a Ortodontia, Cirurgia, Dentistica, Periodontia, Desordem Temporo-mandibular (DTM) e Endodontia (Quadro 1). 
Quadro 1 - Distribuição das referências dos artigos selecionados por autor, ano de publicação, país, área de atuação do respectivo artigo, objetivo, amostragem e resultados e conclusão, $\mathrm{n}=10$. Maceió - $\mathrm{AL}, 2019$.

\begin{tabular}{|c|c|c|c|c|}
\hline $\begin{array}{c}\text { Autor } \\
\text { (Ano)/País }\end{array}$ & $\begin{array}{l}\text { Área de } \\
\text { atuação }\end{array}$ & Objetivo & Amostragem/ Resultados & Conclusão \\
\hline $\begin{array}{l}\text { (ANG } \text { KHAW } \\
\text { CM, et al., } \\
2018) \\
\text { Austrália }\end{array}$ & Ortodontia & $\begin{array}{l}\text { O objetivo da análise na cavidade oral foi } \\
\text { determinar a atuação do laser através da } \\
\text { divisão em dois grupos tendo como } \\
\text { principal objetivo investigar o efeito da } \\
\text { terapia a laser de baixo nível (LLLT) no } \\
\text { tratamento de reparos na reabsorção } \\
\text { radicular inflamatória induzida por } \\
\text { ortodontia (OIIRR). }\end{array}$ & $\begin{array}{l}\text { Vinte pacientes foram incluídos neste estudo, com } 1 \\
\text { lado designado aleatoriamente para receber LLLT, e } \\
\text { o outro lado serviu como uma farsa. / Não foi } \\
\text { estabelecido resultados relevantes nos pacientes } \\
\text { tratados com LLLT e o grupo } \\
\text { Controle. }\end{array}$ & $\begin{array}{l}\text { Não foi possível encontrar Nenhuma } \\
\text { diferença significativa entre os grupos } \\
\text { LLLT e controle falso no reparo OIIRR. }\end{array}$ \\
\hline $\begin{array}{l}\text { (ÜRETÜRK SE, } \\
\text { et al., 2017) / } \\
\text { Turquia }\end{array}$ & Ortodontia & $\begin{array}{l}\text { objetivo do estudo é determinar os efeitos } \\
\text { da laserterapia de baixo nível no } \\
\text { movimento dentário durante a distalização } \\
\text { canina, avaliando os fluidos gengivais. }\end{array}$ & $\begin{array}{l}15 \text { pacientes foram incluídos neste estudo, dos } \\
\text { quais observou-se que a eficácia do encurtamento } \\
\text { de tempo na movimentação do dente por sua vez } \\
\text { diminuiu o tratamento. }\end{array}$ & $\begin{array}{l}\text { O laser acelera o movimento dentário e } \\
\text { pode reduzir toda a duração do } \\
\text { tratamento. }\end{array}$ \\
\hline $\begin{array}{l}\text { (ASUTAY F, et } \\
\text { al., 2018) / } \\
\text { Turquia }\end{array}$ & Cirurgia & 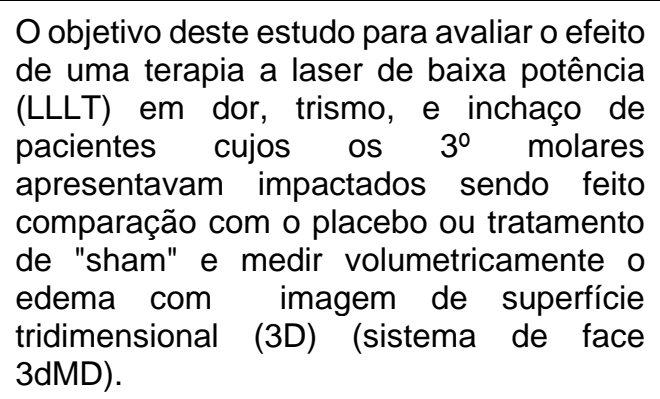 & $\begin{array}{l}45 \text { pacientes foram incluídos neste estudo, dos } \\
\text { quais a LLLT reduziu a dor após extração de } 3^{\circ} \\
\text { molares em dose única, porém não reduziu o } \\
\text { edema facial e o trismo }\end{array}$ & $\begin{array}{l}\text { O LLLT reduziu a intensidade da dor } \\
\text { após cirurgia no terceiro molar em dose } \\
\text { única. Os resultados deste estudo } \\
\text { revelaram que o LLLT reduziu o } \\
\text { inchaço facial, mas não foram } \\
\text { encontradas diferenças na redução do } \\
\text { edema facial e o trismo. }\end{array}$ \\
\hline $\begin{array}{l}\text { MOOSAVI H, } \\
\text { ARJMAND N, } \\
\text { AHRARI F, et } \\
\text { al., 2016) / Irã }\end{array}$ & Dentistica & $\begin{array}{l}\text { O estudo teve como objetivo analisar o } \\
\text { efeito da laserterapia de baixo nível na } \\
\text { sensibilidade dentária induzida pelo } \\
\text { clareamento em consultório. }\end{array}$ & $\begin{array}{l}66 \text { pacientes foram incluídos neste estudo, dos } \\
\text { quais LLLT não reduziu a } \\
\text { sensibilidade imediata ( } 1 \mathrm{~h} \text { depois do } \\
\text { clareamento), mas diminuiu a sensibilidade em } 24 \\
\text { e } 48 \mathrm{~h} \text {. } \\
\text { O grupo } 2 \text { ( } 880 \mathrm{~nm} \text { ) foi significativamente } \\
\text { mais eficaz do que o grupo } 1 \text { ( } 660 \mathrm{~nm}) \mathrm{na} \\
\text { diminuição da sensibilidade ( } 24 \mathrm{~h} \text { após o } \\
\text { clareamento), embora, ambos apresentaram } \\
\text { menor sensibilidade que o grupo placebo (no } \\
\text { intervalo de } 48 \mathrm{~h} \text { ). }\end{array}$ & $\begin{array}{l}\text { O LLLT com laser de diodo } \\
\text { infravermelho pode ser recomendado } \\
\text { como uma estratégia adequada para } \\
\text { reduzir a intensidade da sensibilidade } \\
\text { dentária após o clareamento no } \\
\text { consultório. }\end{array}$ \\
\hline
\end{tabular}

REAS/EJCH | Vol.Sup.n.39 | e2142 | DOI: https://doi.org/10.25248/reas.e2142.2020 Página 5 de 10 


\begin{tabular}{|c|c|c|c|c|}
\hline $\begin{array}{l}\text { HEIDARI M, et } \\
\text { al., 2017) / Irã }\end{array}$ & Periodontia & $\begin{array}{l}\text { O trabalho teve como objetivo avaliar se a } \\
\text { fotobiomodulação a laser (PBM) foi eficaz } \\
\text { em termos de melhorar a cicatrização de } \\
\text { feridas e reduzir a dor pós-operatória. }\end{array}$ & $\begin{array}{l}12 \text { pacientes foram incluídos neste estudo, dos } \\
\text { quais Os resultados mostraram que o } \\
\text { fotobiomodulação a laser com os } \\
\text { parâmetros utilizados neste estudo foi útil na } \\
\text { aceleração da epitelização completa do sítio } \\
\text { doador palatino após a coleta. No entanto, o efeito } \\
\text { do laser no alívio da dor pós-operatória não foi } \\
\text { confirmado. }\end{array}$ & $\begin{array}{l}\text { Os parâmetros utilizados neste estudo } \\
\text { podem acelerar a taxa de epitelização } \\
\text { no local doador. No entanto, não } \\
\text { reduziu a dor pós-operatória. }\end{array}$ \\
\hline $\begin{array}{l}\text { PAMUK F, et } \\
\text { al., 2017) } \\
\text { Turquia }\end{array}$ & Periodontia & $\begin{array}{l}\text { O respetivo trabalho teve como principal } \\
\text { foco avaliar os efeitos da laserterapia de } \\
\text { baixo nível (LLLT) como adjuvante ao } \\
\text { escamação e aplainamento radicular (SRP) } \\
\text { em pacientes fumantes e não fumantes } \\
\text { com periodontite crônica. }\end{array}$ & $\begin{array}{l}60 \text { pacientes foram incluídos neste estudo, dos } \\
\text { quais A LLLT como complemento ao tratamento } \\
\text { periodontal não cirúrgico pode ajudar a resolver } \\
\text { a inflamação e auxilia a cicatrização do tecido } \\
\text { periodontal, particularmente em pacientes com } \\
\text { periodontite crônica }\end{array}$ & $\begin{array}{l}\text { Dentro dos limites deste estudo, pode- } \\
\text { se entender que a LLLT desempenha } \\
\text { um papel na modulação dos níveis do } \\
\text { fluido periodontal gengival, } \\
\text { particularmente em pacientes fumantes } \\
\text { com periodontite crônica, e pode, } \\
\text { portanto, ser recomendada como um } \\
\text { complemento para tratamento } \\
\text { periodontal cirúrgico. }\end{array}$ \\
\hline $\begin{array}{l}\text { (MAGRI LV, et } \\
\text { al., 2018) / } \\
\text { Brasil }\end{array}$ & $\begin{array}{l}\text { desordem } \\
\text { temporo- } \\
\text { mandibular } \\
\text { (DTM) }\end{array}$ & $\begin{array}{l}\text { O objetivo foi analisar os efeitos } \\
\text { inespecíficos (placebo, remissão } \\
\text { espontânea e regressão à média) da } \\
\text { laserterapia de baixa intensidade (LBI) em } \\
\text { mulheres com dor miofascial (desordem } \\
\text { temporomandibular dolorosa (DTM), bem } \\
\text { como diferencie entre respondedores e } \\
\text { clusters de não respondedores de LLLT } \\
\text { ativo e placebo de acordo com os níveis de } \\
\text { ansiedade, cortisol salivar, uso de } \\
\text { contraceptivos orais e período pré- } \\
\text { menstrual. }\end{array}$ & $\begin{array}{l}64 \text { pacientes foram incluídos neste estudo, dos } \\
\text { quais A analgesia promovida pela LLLT em } \\
\text { mulheres com dor miofascial é resultado de } \\
\text { efeitos não específicos durante o período } \\
\text { de tratamento, embora a LLLT seja mais } \\
\text { eficaz na manutenção da analgesia após } \\
\text { o tratamento ( } 30 \text { dias) para o grupo de } \\
\text { mulheres com ansiedade moderada (cortisol } \\
\text { salivar acima de } 10 \mathrm{ng} / \mathrm{ml} \text { e sem uso de } \\
\text { contraceptivos). }\end{array}$ & $\begin{array}{l}\text { Mulheres com ansiedade severa e no } \\
\text { período pré-menstrual não reduziram a } \\
\text { dor com nenhum LLLT. LLLT ativo e } \\
\text { placebo tiveram eficácia semelhante } \\
\text { durante o período de tratamento; no } \\
\text { entanto, mulheres com ansiedade } \\
\text { moderada, níveis de cortisol acima de } \\
10 \mathrm{ng} / \mathrm{ml} \text { e sem uso de contraceptivos } \\
\text { mantêm a analgesia por mais tempo } \\
\text { com LLLT ativo do que com placebo } \\
\text { (acompanhamento de } 30 \\
\text { dias). Mulheres com baixos níveis de } \\
\text { ansiedade, cortisol salivar abaixo de } 10 \\
\mathrm{ng} / \mathrm{ml} \text { e com uso de contraceptivos } \\
\text { apresentaram maior redução da dor. }\end{array}$ \\
\hline
\end{tabular}

REAS/EJCH | Vol.Sup.n.39 | e2142 | DOI: https://doi.org/10.25248/reas.e2142.2020 Página 6 de 10 


\begin{tabular}{|c|c|c|c|c|}
\hline $\begin{array}{l}\text { (MACHADO } \\
\text { BC, et al., 2016) } \\
\text { / Brasil }\end{array}$ & $\begin{array}{l}\text { desordem } \\
\text { temporo- } \\
\text { mandibular } \\
\text { (DTM) }\end{array}$ & $\begin{array}{l}\text { Este estudo investigou a eficácia da } \\
\text { combinação da laserterapia de baixo nível } \\
\text { (LLLT) com exercícios motores orais (OM- } \\
\text { exercícios) para reabilitação de pacientes } \\
\text { com desordens temporomandibulares } \\
\text { crônicas (DTMs). }\end{array}$ & $\begin{array}{l}64 \text { pacientes foram incluídos neste estudo, dos } \\
\text { quais A LLLT com exercícios motores orais foi mais } \\
\text { eficaz reabilitação da DTM que apenas a LLLT. } \\
\text { Resultados semelhantes de tratamento, com } \\
\text { o protocolo de terapia orofacial miofuncional. }\end{array}$ & $\begin{array}{l}\text { O LLLT combinado com os exercícios } \\
\text { de OM foi mais eficaz na promoção da } \\
\text { reabilitação da DTM do que o LLLT } \\
\text { sozinho. Resultados semelhantes de } \\
\text { tratamento foram verificados com o } \\
\text { protocolo OMT. }\end{array}$ \\
\hline $\begin{array}{l}\text { (NABI S, et al., } \\
2018 \text { / I ndia }\end{array}$ & Endodontia & $\begin{array}{l}\text { Avaliar o efeito da irradiação a laser de } \\
\text { baixo nível e do ibuprofeno na redução do } \\
\text { início e da gravidade da dor pós-operatória } \\
\text { após endodontia de visita única. }\end{array}$ & $\begin{array}{l}120 \text { pacientes foram incluídos neste estudo, dos } \\
\text { quais a terapia com laser de baixa intensidade } \\
\text { pode ser uma alternativa eficaz para o uso } \\
\text { convencional de AINE's no controle da dor } \\
\text { pós-endodôntica. }\end{array}$ & $\begin{array}{l}\text { Demonstrou que a terapia a laser de } \\
\text { baixo nível pode ser uma alternativa } \\
\text { eficaz para o uso convencional de anti- } \\
\text { inflamatórios não esteroides no } \\
\text { controle da dor pós-endodôntica, } \\
\text { eliminando assim os efeitos adversos } \\
\text { desses medicamentos nos pacientes. }\end{array}$ \\
\hline $\begin{array}{l}\text { (ARSLAN H, et } \\
\text { al., 2017) / } \\
\text { Malásia }\end{array}$ & Endodontia & $\begin{array}{l}\text { Este estudo avaliou o efeito da LLLT na dor } \\
\text { pós-operatória após o retratamento do } \\
\text { canal radicular (RCR). }\end{array}$ & $\begin{array}{l}32 \text { pacientes foram incluídos neste estudo, dos } \\
\text { quais Nos primeiros } 4 \text { dias, a dor pós-operatória } \\
\text { reduziu significativamente no grupo LLLT em } \\
\text { comparação com o grupo placebo ( } P<0,05) \text {. No } \\
\text { entanto, não foram encontradas diferenças } \\
\text { estatisticamente significantes na dor pós-operatória } \\
\text { entre os dois grupos após } 5 \text { e } 7 \text { dias }(P>0,05) \text {. O } \\
\text { número de pacientes que necessitaram de } \\
\text { analgésicos foi menor no grupo LLLT do que no } \\
\text { grupo placebo }(P<0,05) \text {. Nenhum paciente relatou } \\
\text { dor durante a aplicação da LLLT. }\end{array}$ & $\begin{array}{l}\text { O tratamento utilizando a laserterapia } \\
\text { pode reduzir a dor pós-operatória após } \\
\text { o retratamento do canal radicular dos } \\
\text { molares inferiores. }\end{array}$ \\
\hline
\end{tabular}

Fonte: Neto JMAS, et al., 2019.

REAS/EJCH | Vol.Sup.n.39 | e2142 | DOI: https://doi.org/10.25248/reas.e2142.2020 Página 7 de 10 
$\mathrm{Na}$ atualidade inúmeros artigos referentes ao processo de utilização de Laser de baixa intensidade são encontrados principalmente os que envolvem procedimentos relacionados aos tecidos duros e moles, e muitas especializações odontológicas utilizam tal procedimento para melhorar os resultados pós realização dos procedimentos como: Ortodontia, Cirurgia, Dentistica, Periodontia, Desordem Temporo-mandibular (DTM) e Endodontia dentre tantas outras. No entanto, a amplitude das diversas variedades encontradas no mercado são especificas para determinados tipos de técnicas que irão ser utilizadas, sendo levado como consideração os meios ativos que neles são encontrados além dos parâmetros da dosagem (NABI $S$, et al., 2018).

Existem inúmeros tipos de LLLP sendo utilizados, porém o mais aplicado no cotidiano do CD devido os princípios ativos encontrados nele é o de diodo que possui como comprimento de ondas eletromagnéticas vermelhas $(632,660 \mathrm{~nm})$ e infravermelhos $(820,940 \mathrm{~nm})$. Isso porque o comprimento de onda que possui valores menores não possui resultados satisfatórios pois eles atuam camadas mais superficiais atingindo só ao epitélio, no entanto os que possuem uma melhor performance nos comprimentos de ondas como é o caso do infravermelho, a sua amplitude de atuação é mais profunda (ARSLAN H, et al., 2017).

Dos dez artigos que passaram pela revisão na integra foi observado que, os que são mais utilizados pelo CD são os relacionados a periodontia. Os estudos enfatizam quem o LLLT é um ótimo coadjuvante no tratamento das raspagens radiculares onde os pacientes possuem doenças periodontais, visto que com a utilização do laser e tendo conhecimento dos seus componentes ativos ele ajuda no processo de cicatrização tecidual e diminui as dores devido seu efeito analgésico (PAMUK F, et al., 2017). E mesmo os pacientes que possuíam doenças sistêmicas como diabetes a atuação do agente presente no LLLP minimizaram as dores e melhoraram significantemente o processo de cicatrização devido a minimização de citocinas e periodontopatógenos dos locais onde passaram pela aplicação do laser, no entanto as dores pós operatórias não minimizadas (HEIDARI M, et al., 2017).

No que se refere a ortodontia os resultados dos procedimentos utilizando o LLLT constatou-se um resultado satisfatório nos pacientes que possuíam tropismo celular nos tecidos ósseos, possibilitando o reparo ósseo como no aumento mais significativo da maxila e desenvolvendo da técnica de remodelagem óssea, por causa da sua ação anti-inflamatória e analgésica, acelerando e melhorando a deslocação ortodôntica. A grande preponderância referente a essa temática estabelecera resultados satisfatórios quando se utilizou ondas contínuas de luzes infravermelho, com o princípio ativo de diodo (AlGaAs), com os feixes de luzes na média de 2 a $5 \mathrm{~J} / \mathrm{cm}^{2}$ (joules por centímetro quadrado). No que se refere ao resultado da analgesia, o laser de baixa intensidade demostrou-se bem eficaz, em doseamentos similares, obteve ótimo resultados no conforto dos pacientes logo após a aplicação de separadores ortodônticos (ÜRETÜRK SE, et al., 2017). Quanto ao resultado não satisfatório referente aos mesmos procedimentos feito por Ang Khaw $\mathrm{CM}$, et al. (2018) possivelmente obteve-se devido a utilização da onda eletromagnética vermelha que foi utilizada, do qual obteve uma inferior absorção pela raiz radicular.

Os procedimentos cirúrgicos relacionados aos dentes sisos seja ele incluso ou impactado geralmente ocorre determinados problemas referente ao pós-cirúrgicos sendo os mais recorrentes: Edemas, trismo e dores. Quando se é aplicado o LLLT que possuem ondas eletromagnéticas infravermelho (780 a $910 \mathrm{~nm}$ ) (nanômetro), de maneira continua ou pulsátil, dentre de uma ludicidade que varia em 4 a $7,5 \mathrm{~J} / \mathrm{cm}^{2}$, apresentase eficiente, essencialmente na atenuação das dores pós-operatórias, sendo ocorrido os efeitos analgésicos devido à absorvimento dos feixes de luzes pelos nociceptores, realizando um resultado adstringente a respeito das fibras nervosas, que minimizam a celeridade da condução, restringindo a abrangência das possibilidades de ação dos compostos, o que extingui o processo inflamatório neurogênico. Assim sendo, o laser de baixa intensidade agiliza o estabelecimento de modificações neurossensoriais em indivíduos que são sujeitos à osteotomia sagital bilateral (ASUTAY F, et al., 2018).

Ao ser analisado a importância do LLLT nas disfunções temporomandibulares (DTM), observa-se na maioria dos casos como sendo um tratamento complementar. Nos respectivos estudos feitos através das literaturas citadas foi visto que, quando se utilizou o laser obteve resultados parecidos com os placebos utilizados (MAGRI LV, et al., 2018). 
Já quando o tratamento é correlacionado a alguns exercícios relacionados as funções orofaciais diminuem o processo doloroso da DTM, devido a presença dos fatores analgésicos que são encontrados no LLLT (MACHADO BC, et al., 2016).

O uso do LLLT no tratamento endodôntico demostra ser uma boa alternativa sendo utilizados com AINEs (antiinflamatóriso não esteroides) onde ocasiona a diminuição de dores pós-endodontia, suprimindo os resultados indesejáveis de tais substancias utilizadas tanto no tratamento endodôntico como também no retratamento do mesmo (NABI S, et al., 2018) e (ARSLAN H, et al., 2017).

O artigo relacionado a dentística enfatizou a utilização do LLLT no tratamento pois a realização do procedimento clareador feito no consultório odontológico, utilizou um feixe de luz no comprimento de 660 e $810 \mathrm{~nm}$. Sendo observado resultado satisfatório a dessensibilização, no entanto o infravermelho apresentou um potencial mais satisfatório que o LLLT de luz vermelha (MOOSAVI H, et al., 2016).

Para a obtenção de uma pesquisa mais esclarecedora referentes as grandes áreas de especializações odontológicas relacionando o LLLT e seus benefícios, uma das limitações seria a escassez de estudos mais detalhados sobre essa temática relacionado a níveis comparativos, características estas que deverá ser levado em consideração na elaboração de novas pesquisas.

\section{CONSIDERAÇÕES FINAIS}

Ao ser feito uma aprofundada revisão sistemática dos artigos com o objetivo de obter qualidade científica do respectivo assunto, observou-se que a utilização dos lasers é uma das ferramentas mais indispensáveis no uso odontológico, constatando-se que os processos de laserterapias que possuem baixa intensidade apresentam imensas aplicações e indicações para os Cirurgiões Dentistas, principalmente por apresentarem individualidades no que se refere a coerência, monocromaticidade, direcionamento e unidirecionalidade dos feixes de luz, sendo bastante importante nos tratamentos coadjuvantes, obtendo-se um tratamento bastante confiável nas mais diversas especializações odontológicas, onde não possui efeitos colaterais decorrentes, sendo de fundamental importância a aptidão do profissional ao usar essa tecnologia.

\section{REFERÊNCIAS}

1. ALSAYED HASAN MMA, et al. Low-level laser therapy effectivenes in accelerating orthodontic tooth movement: $A$ randomized controlled clinical trial. Angle Orthodontist, v. 87, n.4, 2017. p. 499-504.

2. ANG KHAW CM, et al. Physical properties of root cementum: Part 27. Effect of low-level laser therapy on the repair of orthodontically induced inflammatory root resorption: A double-blind, split-mouth, randomized controlled clinical trial. American Journal of Orthodontics and Dentofacial Orthopedics, v.3, n.154, 2018. p. 326 -336.

3. ARSLAN H, et al. Effect of Low-level Laser Therapy on Postoperative Pain after Root Canal Retreatment: A Preliminary Placebo-controlled, Triple-blind, Randomized Clinical Trial. Journal of Endodontics, v. 43, n. 11, 2017, p.1765-1769.

4. ASUTAY F, et al. Three-dimensional evaluation of the effect of low-level laser therapy on facial swelling after lower third molar surgery: A randomized, placebo-controlled study. Nigerian Journal of Clinical Practice, v. 21, n. 9, sep. 2018, p.1107-1113.

5. CIEPLIK F, et al. Antimicrobial photodynamic therapy - what we know and what we don't.Critical Reviews in Microbiology, v.44, n.5, p. 571-589, sep. 2018.

6. FARIAS RD, et al. Evaluation of the use of low-level laser therapy in pain control in orthodontic patients: A randomized split-mouth clinical trial. Angle Orthodontist, v. 86, n.2, 2016. p. 193-198.

7. HEIDARI M, et al. Effect of laser photobiomodulation on wound healing and postoperative pain following free gingival graft: A split-mouth triple-blind randomized controlled clinical trial. Journal of Photochemistry \& Photobiology, B: Biology, v. 172, 2017, p.109-114.

8. MACHADO BC, et al. Effects of oral motor exercises and laser therapy on chronic temporomandibular disorders: a randomized study with follow-up. Lasers in Medical Science, v. 31, n. 5, 2016, p.945-954.

9. MAGRI LV, et al. Non-specific effects and clusters of women with painful TMD responders and non-responders to LLLT: double-blind randomized clinical trial. Lasers in Medical Science, v. 32, n. 2, 2018, p.385-392. 
10. MOOSAVI H, et al. Effect of low-level laser therapy on tooth sensitivity induced by in-office bleaching. Lasers in Medical Science, v. 31, n. 4, 2016, p.713-719.

11. NABIS, et al. Effect of preoperative ibuprofen in controlling postendodontic pain with and without low-level laser therapy in single visit endodontics: A randomized clinical study. Indian Journal of Dental Research, v. 29, n. 1, 2018, p.46-50.

12. PAMUK F, et al. The effect of low-level laser therapy as an adjunct to non-surgical periodontal treatment on gingival crevicular fluid levels of transforming growth factor-beta 1, tissue plasminogen activator and plasminogen activator inhibitor 1 in smoking and nonsmoking chronic periodontitis patients: A split-mouth, randomized control study. Journal of Periodontal Research, v. 52, n. 5, 2017, p.872-882.

13. POL R, et al. Efficacy of Anti-Inflammatory and Analgesic of Superpulsed Low Level Laser Therapy After Impacted Mandibular Third Molars Extractions. Journal of Craniofacial Surgery, v. 27, n.3, 2016. p.685-690.

14. SANT'ANNA EF, et al. High-intensity laser application in Orthodontics. Dental Press Journal of Orthodontics, v. 22 , n.6, 2017.p. $99-109$.

15. SHIMTOME LK, et al. Avaliação clinica da laserterapia no tratamento de hipersensibilidade dentinária. ciência Odontológica brasileira, 10 (1); 2007, p. 26-33.

16. SRIVASTAVA VK, MAHAJAN S. Diode lasers: a magical wand to an orthodontic practice. Indian Journal of Dental Research, v. 25, n.1, 2014, p.78-82.

17. TORKZABAN $P$, et al. Low-level laser therapy with $940 \mathrm{~nm}$ diode laser on stability of dental implants: a randomized controlled clinical trial. Lasers in Medical Science, v.33, n. 2, 2018, p.287-293.

18. ÜRETÜRK SE, et al. The effect of low-level laser therapy on tooth movement during canine distalization. Lasers in Medical Science, v. 32, n.4, 2017. p. 757-764. 